\title{
Challenges Faced by US-Trained Gastroenterologists When Pursuing a Medical Career Outside of the USA
}

\author{
Louis Chaptini ${ }^{1,2}$
}

Published online: 24 October 2017

(C) Springer Science+Business Media, LLC 2017

\section{Introduction}

Although the vast majority of US-trained medical practitioners choose to practice in the USA, there are circumstances in which US-trained gastroenterologists choose to pursue a career outside the USA. A common scenario involves physicians with foreign medical degrees who complete residency and fellowship in the USA and decide to return to their home country or to an area that more closely resembles their cultural background. While the majority of these foreign medical graduates stay and pursue a career in the USA, familial and cultural motives as well as immigration obstacles (inability to find a path to permanent residency in the USA) are the main causes underlying the decision to leave. The latter may become a more common trigger now that new regulations have been imposed on the H1 visa program, a pathway used by most foreign physicians to convert their visa status in order to be able to practice in the USA.

There are other situations in which US-trained gastroenterologists choose to practice abroad, such as looking for a special experience, to take advantage of opportunities not commonly available in the USA, to obtain better compensation, and to volunteer in underserved areas of the world.

In recent years, global healthcare has experienced changes with resultant increased standardization of medical

Louis Chaptini

louis.chaptini@yale.edu

1 Department of Internal Medicine/Gastroenterology, American Hospital Dubai, Dubai, United Arab Emirates

2 Division of Gastroenterology, Department of Medicine, Yale University School of Medicine, New Haven, CT, USA practice similar to the impact that globalization has had on many major industries. Nonetheless, when practicing in a foreign, unfamiliar environment, physicians should take into consideration several important professional and cultural facets associated with such a major career decision. The purpose of this article is to shed light on some of the differences encountered when practicing medicine in general and gastroenterology specifically in different parts of the world.

\section{Documentation and Certification Requirements}

In general, the practitioner seeking to practice in another country should anticipate major delays in getting established due to documentation and certification requirements of varying stringency. These comprise a wide range of items including visa requirements (for those not returning to their home countries), medical licensure requirements, and registration and certification of medical specialty degrees in the host country (with the possibility of sitting for additional special examinations to be able to practice). Although US board-certified physicians are in general in demand worldwide, some countries are more protective of their own physicians, requiring considerable training and certifications prior to joining their ranks. For example, physicians wishing to practice in any capacity in the UK must be registered and licensed to practice with the General Medical Council and must fulfill a host of professional requirements, demonstrating their medical knowledge and skills, and in some cases by sitting for an assessment test board. In New Zealand, new registrants must be supervised for at least their first 12 months in order to become culturally acclimated. In the United Arab Emirates, a US board-certified physician can register to work as a 
"consultant" without having to sit for any medical knowledge assessment examinations, whereas physicians from certain other countries have to register as "specialists" (lesser privileges than consultants) and pass written and oral examinations to demonstrate their medical knowledge and skills.

Physicians who hold US citizenship or permanent residency and are keeping their ties with the USA should also assess the implications of an overseas career on their taxes, life and disability insurance, and retirement plans. Unlike most other countries, expatriates from the USA are still required to pay income taxes to their home countries, although part of their income will be tax-exempt.

\section{Cultural Considerations}

Despite the observation that English has become the de facto global standard language of healthcare, this may not be the case at the local population level. Practicing in a country where English is not the primary language may add another layer of complexity as translation services relying on trained staff or technology (over the phone interpretation services) are not widely available.

Communicating with healthcare professionals and staff may be challenging. At a language level, although English is widely used among healthcare professionals, one may have to get used to different accents, sometimes very hard to understand. Furthermore, instruments and equipment may be named differently or simply be different from what one is familiar with. At a relationship level, hierarchy considerations can be very important in shaping interactions in the workplace. Compared to Western countries such as the USA and Canada, many countries have a stricter hierarchical structure that can have an impact on the professional interactions. For example, although providing unsolicited opinions, comments, or challenges for unprofessional behavior or medical mistakes on the part of an individual of higher rank or position is acceptable and encouraged in the USA, it may be scarcely tolerated in some Asian cultures.

Communicating with patients is equally subject to the same cultural considerations. Translation services provided when English is not the primary language can be timeconsuming, cumbersome, and of variable quality, depending almost entirely on the competence of the individual providing this service. When dealing with patients, using translation services affects many aspects of the doctorpatient interaction ranging from simple history taking to discussing management options and recommendations to the more complex tasks of explaining procedures and obtaining informed consents. When, as commonly occurs, family members offer their assistance, asking for an independent translator may not be culturally acceptable.

Language barrier aside, one should then be sensitive to the patients' perceptions and understanding of disease and the presence of taboo subjects. For instance, in many Middle Eastern countries, family members of patient diagnosed with cancer will often ask the physician to refrain from disclosing the diagnosis to the patient. Subjects such as sexually transmitted diseases are not easily approached in some contexts. For instance, advising vaccination against human papilloma virus (HPV) (as is generally recommended) to a teenager for whom biologic treatment for Crohn's disease is prescribed may be shocking to the patient and her parents.

\section{Medical Practice Differences}

Although practice guidelines issued by reputable societies are a major influence on how US-trained physicians practice medicine, these may not apply in other environments where resources, risk factors, and disease prevalence are different. For example, US guidelines for colon cancer screening are not necessarily implemented in other parts of the world for multiple reasons, including lack of data to support their benefit, insufficient resources (specialists, equipment), and lack of awareness and acceptance among the public. The concept of screening is hard to accept in many cultures, especially when screening involves an invasive procedure such as colonoscopy. Even when resources are available and health authorities recommend screening, these efforts can be met with resistance at the individual level. Conversely, US-trained physicians are unfamiliar with some guidelines driven by the higher prevalence of diseases in some countries such as screening upper endoscopy for gastric cancer in some Asian countries.

In countries where resources are limited, physicians may find themselves deviating from generally accepted standards-of-care. Many gastroenterology practices around the world lack the training and/or the equipment to perform banding in cases of variceal bleeding, resorting instead to sclerotherapy, a procedure less familiar to young US practitioners and falling out of favor in the USA. Endoclips may not be available to use for the treatment of ulcer bleeding or to close a mucosal defect after polypectomy. The latest generation of endoscopes may not be available, which may create a hindrance to someone accustomed to narrow-band imaging and high-definition images.

In places where medical tourism is widespread, physicians may find themselves administering tests that are not indicated as part of a medical package offered by their organization. Some of these "wellness packages" include 
items not conforming to US-based guidelines such as fecal occult blood testing at age 40 or routine abdominal ultrasound and liver tests in asymptomatic patients.

Expertize in gastrointestinal subspecialties such as motility disorders is generally not available outside of urban quaternary care centers, clearly impacting the ability to treat patients with pelvic dyssynergia and esophageal motility disorders. The same applies to pancreatic and biliary expertize with limited availability in certain areas of advanced endoscopic procedures such as endoscopic ultrasound (EUS) and endoscopic retrograde cholangiopancreatography (ERCP). For US physicians coming from centers where subspecialty services are readily available, this can create an additional layer of frustration.

Medications, particularly expensive ones such as biologics used for inflammatory bowel diseases and directacting antivirals used for hepatitis $\mathrm{C}$, may not be available in certain countries or may not be covered by insurance.

Disease prevalence may be different depending on geographical location. For instance, hydatid cysts are rarely encountered in the USA but are prevalent in parts of the Middle East and should always be on the differential in patients with hepatic cysts. Helicobacter pylori strains may have different resistance profile to antibiotics, which affects the choice of first-line therapy.

\section{Personal Considerations}

On a personal level, the decision to move abroad should not be taken lightly. From a career perspective, practicing abroad may lead to a major professional shift with possible impact on future plans. For instance, if one leaves an academic position in the USA with a goal to return to a similar position after few years abroad, it is advisable to keep connections as volunteer faculty with an academic institution in the USA or join an academic center abroad. Furthermore, one should ensure that all skills, particularly the ones acquired through extra training, are frequently used abroad as these may be easily lost, especially with the rapid advancement of technology.

Physicians moving with their families have to consider several important factors that will affect the comfort and well-being of all family members. The spouse should explore job opportunities abroad, especially if they were part of the workforce in the USA. One should investigate the different schooling systems and the availability of schools offering American curricula, especially if planning to move back to the USA. It is important to contemplate how well the family will adapt to the new country and set realistic expectations. It typically takes time to adjust to all aspects of the new environment from simple components such as different weather and food, to more complicated ones such as divergent cultural backgrounds and unfamiliar types of social interactions.

\section{Conclusion}

In summary, pursuing a medical career outside the USA can be exciting, inspiring, and at the same time complex and challenging. Multiple aspects of such a move should be carefully planned and studied to avoid unexpected surprises and ensure success. 